\title{
BMJ Open Impact of universal health coverage on urban-rural inequity in psychiatric service utilisation for patients with first admission for psychosis: a 10-year nationwide population-based study in Taiwan
}

\author{
Chih-Lin Chiang, ${ }^{1,2}$ Pei-Chun Chen, ${ }^{3}$ Ling-Ya Huang, ${ }^{4}$ Po-Hsiu Kuo, ${ }^{3}$ \\ Yu-Chi Tung, ${ }^{5}$ Chen-Chung Liu, ${ }^{6,7}$ Wei J Chen ${ }^{1,3,6}$
}

To cite: Chiang C-L, Chen P-C, Huang L-Y, et al. Impact of universal health coverage on urban-rural inequity in psychiatric service utilisation for patients with first admission for psychosis: a 10-year nationwide population-based study in Taiwan. BMJ Open 2016;6: e010802. doi:10.1136/ bmjopen-2015-010802

- Prepublication history and additional material is available. To view please visit the journal (http://dx.doi.org/ 10.1136/bmjopen-2015010802).

Received 7 December 2015 Revised 3 February 2016 Accepted 12 February 2016

CrossMark

For numbered affiliations see end of article.

Correspondence to Dr Wei J Chen; wjchen@ntu.edu.tw

\section{ABSTRACT}

Objective: To examine the disparities in psychiatric service utilisation over a 10-year period for patients with first admission for psychosis in relation to urbanrural residence following the implementation of universal health coverage in Taiwan.

Design: Population-based retrospective cohort study. Setting: Taiwan's National Health Insurance Research Database, which has a population coverage rate of over $99 \%$ and contains all medical claim records of a nationwide cohort of patients with at least one psychiatric admission between 1996 and 2007.

Participants: 69690 patients aged 15-59 years with first admission between 1998 and 2007 for any psychotic disorder.

Main exposure measure: Patients' urban-rural residence at first admissions.

Main outcome measures: Absolute and relative inequality indexes of the following quality indicators after discharge from the first admission: all-cause psychiatric readmission at 2 and 4 years, dropout of psychiatric outpatient service at 30 days, and emergency department (ED) treat-and-release encounter at 30 days.

Results: Between 1998 and 2007, the 4-year readmission rate decreased from $65 \%$ to $58 \%$, the 30 -day dropout rate decreased from $18 \%$ to $15 \%$, and the 30-day ED encounter rate increased from $8 \%$ to $10 \%$. Risk of readmission has significantly decreased in rural and urban patients, but at a slower speed for the rural patients $(p=0.026)$. The adjusted HR of readmission in rural versus urban patients has increased from $1.00(95 \% \mathrm{Cl} 0.96$ to 1.04) in 19982000 to $1.08(95 \% \mathrm{Cl} 1.03$ to 1.12$)$ in $2005-2007$, indicating a mild widening of the urban-rural gap. Urban-rural differences in 30-day dropout and ED encounter rates have been stationary over time.

Conclusions: The universal health coverage in Taiwan did not narrow urban-rural inequity of psychiatric service utilisation in patients with psychosis. Therefore,

\section{Strengths and limitations of this study}

This is the first study to describe the long-term course of mental health inequity in the context of universal health coverage in Taiwan. A nationwide cohort of patients with first admission for psychosis within 10 years was analysed, and service utilisations up to 4 years from discharge were examined.

- Some socioeconomic or disease variables are absent or incomplete in the administrative database. A key variable in this study, patients' residence, is estimated by using a verified algorithm based on patients' insurance classification, insurance registration and location of hospital visit.

- The analyses only include patients who had been admitted to the psychiatric wards for the management of psychosis, so the findings might not be generalised to patients with other nonpsychotic mental illnesses or psychotic patients treated as outpatients throughout their illness course.

other policy interventions on resource allocation, service delivery and quality of care are needed to improve the outcome of rural-dwelling patients with psychosis.

\section{INTRODUCTION}

Psychosis refers to a group of serious mental illnesses characterised by disturbances in reality testing, such as delusion, hallucination, disorganised behaviours and impaired insight. It is regarded as the most severe form of mental illness, affecting 2-3\% of people in their lives, ${ }^{1}$ with the onset peaking 
in late adolescence and early adulthood. Without timely and appropriate treatment, patients usually suffer from long-term, sometimes permanent disabilities. A number of disease-related intrinsic (eg, impaired insight, suspiciousness, agitation, negative symptoms, cognitive deficits) and extrinsic factors (eg, inadequate family supports, stigma, social unawareness) prevent patients from seeking essential mental health care. Service scarcity, inequity and insufficiency further hinder patients from successful recovery. ${ }^{2}$

With universal health coverage (UHC), a priority goal set by the WHO in recent years, there is high expectation that all people, regardless of their socioeconomic status, can obtain health services that they really need without obstacles. ${ }^{3}$ Equity-oriented monitoring across different dimensions, including urban-rural residence, is therefore of paramount importance to the realisation of UHC. ${ }^{4}$ Such equity should be achieved in the management of infectious diseases, major noncommunicable diseases, and maternal and child health issues, as stressed by the WHO, as well as in the management of mental illnesses whose importance in public health has often been ignored. ${ }^{5}$ While the global burden of mental illnesses is ever increasing, ${ }^{6}$ in low income and middle income countries treatment gaps for mental illnesses are still large. ${ }^{7}$ Even in advanced countries like Canada, with a well-established publicly funded health system for decades, mental health service accessibility in socioeconomically deprived areas continues to be a major problem..$^{8-11}$

Several studies have revealed a pre-existing urbanrural inequity in Taiwan's mental health before 1995. In a rapidly transforming society, rural people have faced a myriad of stressors that contributed to the significant urban-rural difference in the prevalence of common mental disorders, such as dissolution of traditional values, worse economic conditions and low education levels. ${ }^{12}$ While the prevalence rates of psychosis in rural and urban areas were similar, ${ }^{12}$ rural patients with psychosis had much fewer inpatient and outpatient resources than urban patients. ${ }^{13}$ The elderly in rural areas utilised less health services than did their counterparts in urban areas in Taiwan. ${ }^{14}$ There were also urban-rural differences in people's attitude towards modern medicine ${ }^{15}$ and patients' capability of affording psychiatric inpatient service. ${ }^{16}$ Presumably, some aspects of urban-rural inequity, especially patients' service availability and affordability, could be amended by a well-established UHC.

Taiwan launched in 1995 its National Health Insurance (NHI), a high-performing single-payer healthcare system which has fulfilled the WHO's definition of UHC by its great financial risk protection and high insurance coverage rate (over 96\% since 1996; over 99\% since 2004). ${ }^{17}$ As one of the most equalitarian health systems, it resembles the Canadian health system in many aspects but has additional features such as very low out-of-pocket payments (since the prescription drugs are also covered) and short waiting times for the patients. Following its implementation, disadvantaged people previously excluded from any insurance plan, who tended to be older and less educated, have had significantly improved access to healthcare. ${ }^{18}$ In addition, healthcare utilisation has become well determined by health needs. ${ }^{19}$ Previous studies in China indicated that the expansion of health coverage and financial protection in rural populations improved their self-perceived psychological health, ${ }^{20}{ }^{21}$ and we wonder if a more comprehensive and barrier-free healthcare system like Taiwan's NHI could render rural patients with psychosis essential mental healthcare similar to what urban patients could obtain.

In the current study, we used nationwide claims data sets to examine the longitudinal evolution of urbanrural inequity indexes during the first decade of NHI implementation. We limited the analyses to patients in the early course of psychosis (ie, with first admission for psychosis) because effective treatments during this critical period may exert the greatest therapeutic impacts. Three quality indicators that were related to health service use were examined for urban-rural inequity: readmission rates (up to 4 years), loss to psychiatric outpatient follow-up at 30 days (a.k.a. early dropout), and all-cause treat-and-release encounter to the emergency department at 30 days (a.k.a. early ED encounter). Readmission, which closely corresponds to psychotic relapses, is a validated outcome indicator of psychosis. ${ }^{22}$ Early dropout and ED encounter in the first 30 days of discharge are also frequently examined quality indicators; the former reflects psychiatric patients' adherence to treatment and subsequent health outcomes, ${ }^{23}$ and the latter reflects inadequate transition of care. ${ }^{24}$ Our hypothesis is that in the first decade of NHI, there would be a trend towards reduction of urban-rural inequity in all or some of the selected quality indicators of psychosis treatment.

\section{METHOD}

\section{Design and data source}

This is a population-based retrospective cohort study using the National Health Insurance Research Database (NHIRD), which includes the claim records of ambulatory care, inpatient care and drug prescriptions of all NHI-enrolled beneficiaries in Taiwan since 1996. In this study, we analysed the 1996-2007 Psychiatric Inpatient Medical Claims Database (PIMCD), consisting of all claim records between 1995 and 2011 of 187113 individuals who had been admitted to Taiwan's psychiatric wards between 1996 and 2007.

\section{Cohort eligibility}

Individuals were eligible if they had been admitted to Taiwan's NHI-based psychiatric wards between 1 January 1996 and 31 December 2007, aged 15-59 years, with a principal inpatient diagnosis of psychosis, including the 
following International Classification of Diseases, Ninth Revision, Clinical Modification codes: schizophrenia (295.x), affective psychosis (296.04, 296.14, 296.24, 296.34, 296.44, 296.54, 296.64), delusional disorder (297.x), substance-induced psychosis (291.3x, 291.5x, 292.1x) and other non-organic psychosis (298.x). Each individual's first admission to receive an inpatient diagnosis of psychosis was identified, and then those whose first admission for psychosis was between 1995 and 1997 were excluded from subsequent analyses because their lifetime first admission for psychosis might be earlier than the launch of NHI. We further excluded patients who were aged less than 15 years at the first admission for psychosis $(\mathrm{N}=440)$ so as to be in accord with the lower age limitation of several previous epidemiological studies of first episode psychosis. ${ }^{25-27}$

\section{Independent variable and covariates}

The independent variable, patients' urban-rural residence, was ascertained in two steps. In the first step, patients' township-level residence was estimated using a validated algorithm, ${ }^{28}$ in which patients' insurance classification, insurance registration, and location of hospital visits for mental illnesses and minor medical or surgical illnesses were taken into consideration. In the second step, the township-level residence was further dichotomised into urban (urbanicity level ${ }^{29}$ one to three) or rural (urbanicity level four to seven), following a previous study investigating urban-rural disparity of service utilisation in Taiwan. ${ }^{30}$

Covariates included insurance amount, calendar year of first admission for psychosis, diagnosis at first admission, length of first admission and hospital type. All these covariates are important predictors of psychiatric readmissions in Taiwan. ${ }^{31}$ Detailed descriptions of the independent variable and covariates are provided in online supplementary section A1.

\section{Indexes of mental health inequity}

Since the discharge from first admission for psychosis, eligible individuals had been followed in the PIMCD until the end of the database (ie, 31 December 2011). Three types of postdischarge adverse events were investigated: all-cause psychiatric readmission (up to 4 years), dropouts (at 30 days) and ED encounters (at 30 days).

Within each time period (admitted in 1998-2000, 2001-2004 or 2005-2007), separate Kaplan-Meier survival functions were established on the basis of different time-to-event information (first readmission/outpatient visit/ED encounter), censored by either the end of the database, dropping out from the database because of death or incarceration, or in the cases of outpatient visit and ED encounter, a readmission episode, whichever came first. The survival or failure probabilities to the adverse event and its 95\% CIs at 24 and 48 months, respectively, in each cohort were presented.

In the final step, the urban-rural disparity of the probability of each type of adverse events was generated as an index of mental health equity in each time period. Both absolute and relative urban-rural disparities, that is, risk difference and risk ratio, were presented according to the recommendations, ${ }^{4}$ with the reference group being the urban cohort.

\section{Statistical analyses}

Pearson's $\chi^{2}$ tests were used to examine the homogeneity of patients' demographic profiles in different time periods. Wilcoxon trend tests were used to examine the presence of temporal trend of each adverse event. The formulae of unadjusted risk difference and risk ratio are provided in online supplementary section A2. To estimate the adjusted risk ratios, first multivariable-adjusted HRs were estimated via Cox proportional hazards modelling. Adjusted covariates included age (10-year age band, starting from 10 to 19 years), gender, time period, economic status, diagnosis, length of first admission and hospital type. Missing values were treated as a separate category of the covariate in the model. Potential interactions between time and residence were also explored. Then the adjusted HR (in the case of readmission or ED encounter) or the reciprocal of the adjusted HR (in the case of loss to outpatient follow-up) was used to stand for the adjusted risk ratio, so that a risk ratio greater than one in such a scenario always means that the rural cohort had a greater risk of developing the adverse event than the urban cohort did.

We performed several sets of sensitivity analyses. In the first analysis, we narrowed the cohort by excluding long-stay patients (length of stay $>180$ days) because the unusual lengths of their first admissions raised the possibility that their genuine lifetime first admissions might be earlier than NHI. In the second analysis, an alternative definition of the probability, that is, without the consideration of censorship, was used to estimate the risk difference and risk ratio, because the Kaplan-Meier estimates are actually conditional probabilities instead of absolute ones. The unadjusted risk difference and risk ratio were estimated via the same formulae, and the multivariable-adjusted risk ratios were estimated via the logistic regression modelling.

All analyses were performed using SAS software V.9.2 (SAS Institute Inc, North Carolina, USA). A two-sided $\mathrm{p}$ value of less than 0.05 for main effects or 0.10 for interaction was considered statistically significant.

\section{RESULTS}

\section{Patients with first admission for psychosis}

From 1998 to 2007, we identified 69690 individuals with a first admission for psychosis (for the flow chart, see online supplementary figure S1), with a median follow-up of 8.3 years (IQR: 5.0 years). The most common reason for censorship was the end of the database. Missing information was very low $(<0.05 \%)$, except for the information of insurance amount (in 1444 
individuals, or $2.1 \%$ of the cohort). Heterogeneities in the distributions of patients' gender, age at first admission, hospital type, length of first admission, diagnosis and economic status across the three time periods were noted (table 1). The distributions in urban or rural residence were not different across the three time periods. Compared with the patients in the period 1998-2000, patients in more recent periods were more likely to be female, admitted at an older age, more likely to be admitted to regional hospitals, having shorter hospital stays, more likely to receive the diagnosis of affective psychosis rather than schizophrenia during the first admissions and more likely to be in the lowest income group. The median interval from the first contact with psychiatric service to the first admission for psychosis, a proxy measure for patients' duration of illness, increased from 1.7 years in 1998-2000 to 2.7 years in 2005-2007. In each period, the interval did not differ significantly between urban and rural patients (see online supplementary table S1).

\section{Risk of readmission at 2 and 4 years}

A total of 41431 patients $(59.5 \%)$ had at least one readmission within 48 months before censorship. Table 2 shows the Kaplan-Meier probabilities of readmission risk at 24 and 48 months, as well as an unadjusted risk difference and risk ratio in each time period. Both the absolute and relative measures of disparities suggested that urban-rural disparities in readmission risks were only marginal in 1998-2000, but had been increasing thereafter. After adjusting for other covariates, a significant interaction between patients' residence and time period was still present (Wald's $\left.\chi^{2}=4.96, \mathrm{df}=1, \mathrm{p}=0.026\right)$.

\section{Risk of dropout and ED encounter at $\mathbf{3 0}$ days}

A total of 54264 patients $(77.9 \%)$ had at least one psychiatric outpatient visit within 30 days before censorship. As shown in table 3, the Kaplan-Meier probabilities of dropout at 30 days had been progressively decreasing in the urban and rural patients. Changes in the magnitude of unadjusted risk ratio suggest that urban-rural disparity in early dropout had been fluctuating during the observation period. After adjusting for other variables, there was no statistically significant change of urbanrural disparity in early dropout risk over time (Wald's $\left.\chi^{2}=1.11, \mathrm{df}=1, \mathrm{p}=0.29\right)$.

A total of 5825 patients $(8.4 \%)$ had at least one ED encounter within 30 days before censorship. As shown in table 3, the Kaplan-Meier probabilities of early ED encounters had been increasing in the urban and rural patients. The unadjusted risk difference between the urban and rural cohorts was small. Although the unadjusted risk ratio appeared to move closer to 1 in the later time period, there was no statistically significant change of urban-rural disparity in early ED encounter risk over time (Wald's $\chi^{2}=2.05, \mathrm{df}=1$, $\mathrm{p}=0.15)$.
Figure 1 is a graphic presentation of the above information. In urban and rural cohorts, readmission (figure 1A) and early dropouts (figure 1B) had decreased and early ED encounters (figure 1C) had increased. While there were no statistically significant changes in urbanrural difference in early dropouts and early ED encounters over time, there was a statistically and clinically significant urban-rural difference in readmission risk over time: the adjusted HR for the rural versus urban patients in readmission at 48 months increased from $1.00(95 \%$ CI 0.96 to 1.04 ) in $1998-2000$ to 1.08 (95\% CI 1.03 to 1.12) in 2005-2007.

Additional sensitivity analyses did not change these findings. See online supplementary table S2-S4 for the details of multivariate Cox $\mathrm{PH}$ models.

\section{DISCUSSION}

This study examined the urban-rural inequity in psychiatric service utilisation since 1998, 3 years after the introduction of NHI in Taiwan. Between 1998 and 2007, readmissions and early dropouts had progressively become less frequent in urban and rural patients in Taiwan following the first admission for psychosis. However, readmissions had decreased faster in urban patients than in rural patients. While the urban-rural inequity in patients' utilisation of outpatient or ED services had been stable, the inequity in readmission, the most important quality indicator in this study, had worsened over time. Nevertheless, the degree of increase in inequity was small. In contrast to our initial hypothesis, Taiwan's NHI, one of the most equalitarian health systems in the world, did not reduce urban-rural inequity in patients' utilisation of hospital care following a first psychosis admission.

\section{Strengths and weakness of this study}

To the best of our knowledge, this is the first study to describe the long-term trajectories of urban-rural inequity in mental health service use in the context of UHC in Taiwan. Other strengths of this study derive from the comprehensiveness of our database. National cohorts encompassing nearly all first-admitted patients each year in Taiwan over a long period were analysed, and the follow-up period for each year's cohort was long enough to generate information regarding patients' 4-year outcome. Besides, patients with all identifiable psychotic syndromes were included, which minimises the potential bias from the change in the syndromal presentation of psychotic disorders over time. ${ }^{32}$ Further, the authors conducted the analyses in accord with the latest equity-oriented monitoring recommendations in the context of UHC. ${ }^{4}$ Both the absolute and relative measures of inequity were presented, with careful control for the established or potential risk factors for adverse events. All these inequity measures reveal similar patterns of change over time, enhancing the robustness of our findings. 
Table 1 Characteristics of the cohorts of patients with first admission for psychosis in Taiwan, 1998-2007 (N=69 690)

\begin{tabular}{|c|c|c|c|c|}
\hline Variable & $1998-2000(\mathrm{~N}=20$ 901) & 2001-2004 (N=29 908) & 2005-2007 (N=18 881) & p Values* \\
\hline Gender & & & & $<0.0001$ \\
\hline Male & $11774(56.3)$ & $17076(57.1)$ & $10102(53.5)$ & \\
\hline Female & $9127(43.7)$ & $12832(42.9)$ & $8779(46.5)$ & \\
\hline Age at first admission (years) & & & & $<0.0001$ \\
\hline $15-19$ & $1473(7.1)$ & 2057 (6.9) & $1118(5.9)$ & \\
\hline $20-29$ & $6165(30.0)$ & 9067 (30.3) & $5059(26.8)$ & \\
\hline 30-39 & 7038 (33.7) & $8780(29.4)$ & $5372(28.5)$ & \\
\hline $40-49$ & 4438 (21.2) & $6700(22.4)$ & $4623(24.5)$ & \\
\hline $50-59$ & $1787(8.6)$ & 3304 (11.1) & 2709 (14.4) & \\
\hline Hospital type & & & & $<0.0001$ \\
\hline Medical centre & $5111(24.5)$ & 6547 (21.9) & 3849 (20.4) & \\
\hline Regional hospital & $10686(51.1)$ & $16651(55.7)$ & $10402(55.1)$ & \\
\hline District hospital & $5080(24.3)$ & 6627 (22.2) & $4543(24.1)$ & \\
\hline Physician clinic & $17(0.1)$ & $69(0.2)$ & $69(0.4)$ & \\
\hline Length of first admission (days) & & & & $<0.0001$ \\
\hline $1-30$ & 8615 (41.2) & $14653(49.0)$ & $9946(52.7)$ & \\
\hline $31-60$ & $5308(25.4)$ & 7404 (24.8) & $4763(25.2)$ & \\
\hline $61-180$ & 4117 (19.7) & 4779 (16.0) & 2501 (13.3) & \\
\hline$>180$ & $2861(13.7)$ & 3072 (10.3) & $1671(8.9)$ & \\
\hline Diagnosis at first admission & & & & $<0.0001$ \\
\hline Schizophrenia & $17011(81.4)$ & $21453(71.7)$ & $12082(64.0)$ & \\
\hline Affective psychosis & $1521(7.3)$ & $4273(14.3)$ & 3655 (19.4) & \\
\hline Substance-induced psychosis & $720(3.4)$ & $1173(3.9)$ & $566(3.0)$ & \\
\hline Delusional disorder & $426(2.0)$ & $737(2.5)$ & $485(2.6)$ & \\
\hline Other non-organic psychosis & $1223(5.9)$ & $2272(7.6)$ & $2093(11.1)$ & \\
\hline Residence & & & & 0.1320 \\
\hline Urban & $14924(71.4)$ & 21592 (72.2) & $13539(71.7)$ & \\
\hline Rural & $5977(28.6)$ & $8313(27.8)$ & $5340(28.3)$ & \\
\hline Economic status & & & & $<0.0001$ \\
\hline Fully employed & 7087 (34.0) & $9846(34.0)$ & $6161(33.4)$ & \\
\hline Dependent & 5009 (24.0) & $6910(23.9)$ & $4076(22.1)$ & \\
\hline Lowest income & 8767 (42.0) & $12193(42.1)$ & $8197(44.5)$ & \\
\hline
\end{tabular}

Values are numbers (percentage).

Five individuals had missing values for residential information; 39 individuals had missing values for hospital type information; 1444 individuals had missing values for economic information.

${ }^{*} p$ Values for the $\chi^{2}$ tests.

Table 2 Risk for readmission within 48 months of discharge for the cohorts of individuals with first admission for psychosis in Taiwan, 1998-2007 (N=69 690)

\begin{tabular}{|c|c|c|c|c|}
\hline \multirow[b]{2}{*}{ Time/stratum } & \multicolumn{3}{|c|}{ Risk for any readmission within 48 months (95\% Cl) } & \multirow[b]{2}{*}{ p Values } \\
\hline & $1998-2000(\mathrm{~N}=20901)$ & 2001-2004 (N=29 908) & 2005-2007 (N=18 881) & \\
\hline \multicolumn{5}{|l|}{ At 48 months } \\
\hline Full cohort & $0.65(0.65$ to 0.66$)$ & $0.61(0.60$ to 0.61$)$ & $0.58(0.57$ to 0.59$)$ & $<0.0001$ \\
\hline Urban cohort & 0.65 (0.64 to 0.66$)$ & $0.60(0.59$ to 0.60$)$ & $0.57(0.56$ to 0.58$)$ & $<0.0001$ \\
\hline Rural cohort & $0.67(0.65$ to 0.68$)$ & $0.63(0.62$ to 0.64$)$ & $0.61(0.60$ to 0.62$)$ & 0.0001 \\
\hline Risk difference & $0.02(0.00$ to 0.03$)$ & $0.03(0.02$ to 0.04$)$ & $0.04(0.03$ to 0.06$)$ & \\
\hline Risk ratio & 1.03 (1.01 to 1.05$)$ & 1.05 (1.03 to 1.08$)$ & $1.08(1.05$ to 1.11$)$ & \\
\hline \multicolumn{5}{|l|}{ At 24 months } \\
\hline Full cohort & $0.54(0.54$ to 0.55$)$ & $0.51(0.50$ to 0.51$)$ & $0.49(0.48$ to 0.50$)$ & $<0.0001$ \\
\hline Urban cohort & 0.54 (0.53 to 0.54$)$ & $0.50(0.49$ to 0.51$)$ & $0.48(0.47$ to 0.49$)$ & $<0.0001$ \\
\hline Rural cohort & $0.56(0.55$ to 0.57$)$ & $0.53(0.51$ to 0.54$)$ & 0.51 (0.50 to 0.53$)$ & 0.0022 \\
\hline Risk difference & $0.02(0.01$ to 0.04$)$ & $0.03(0.01$ to 0.04$)$ & $0.04(0.02$ to 0.05$)$ & \\
\hline Risk ratio & 1.04 (1.01 to 1.07$)$ & 1.05 (1.02 to 1.08$)$ & $1.08(1.04$ to 1.11$)$ & \\
\hline
\end{tabular}


Table 3 Risk for adverse event within 30 days of discharge for the cohorts of individuals with first admission for psychosis in Taiwan, 1998-2007 (N=69 690)

\begin{tabular}{|c|c|c|c|c|}
\hline \multirow[b]{2}{*}{ Time/stratum } & \multicolumn{3}{|c|}{ Risk for any adverse event within 30 days $(95 \% \mathrm{CI})$} & \multirow[b]{2}{*}{ p Values* } \\
\hline & 1998-2000 ( $=20$ 901) & 2001-2004 (N=29 908) & 2005-2007 (N=18 881) & \\
\hline \multicolumn{5}{|l|}{ Loss to follow-up } \\
\hline Full cohort & $0.18(0.17$ to 0.18$)$ & $0.17(0.17$ to 0.17$)$ & $0.15(0.14$ to 0.15$)$ & $<0.0001$ \\
\hline Urban cohort & $0.16(0.16$ to 0.17$)$ & $0.16(0.16$ to 0.17$)$ & $0.14(0.13$ to 0.14$)$ & $<0.0001$ \\
\hline Rural cohort & 0.21 (0.20 to 0.22$)$ & $0.19(0.19$ to 0.20$)$ & $0.17(0.16$ to 0.18$)$ & $<0.0001$ \\
\hline Risk difference & $0.05(0.04$ to 0.06$)$ & 0.03 (0.02 to 0.04$)$ & $0.03(0.02$ to 0.05$)$ & \\
\hline Risk ratio & 1.29 (1.22 to 1.37$)$ & $1.22(1.15$ to 1.28$)$ & $1.24(1.16$ to 1.34$)$ & \\
\hline \multicolumn{5}{|l|}{ ED encounter } \\
\hline Full cohort & $0.08(0.07$ to 0.08$)$ & 0.09 (0.09 to 0.09$)$ & $0.10(0.10$ to 0.10$)$ & $<0.0001$ \\
\hline Urban cohort & 0.08 (0.08 to 0.09$)$ & 0.09 (0.09 to 0.10$)$ & $0.10(0.10$ to 0.11$)$ & $<0.0001$ \\
\hline Rural cohort & 0.07 (0.06 to 0.07$)$ & 0.08 (0.07 to 0.08$)$ & 0.09 (0.09 to 0.10$)$ & $<0.0001$ \\
\hline Risk difference & $-0.02(-0.02$ to -0.01$)$ & $-0.02(-0.02$ to -0.01$)$ & $-0.01(-0.02$ to 0.00$)$ & \\
\hline Risk ratio & $0.80(0.72$ to 0.90$)$ & $0.83(0.76$ to 0.90$)$ & $0.93(0.85$ to 1.03$)$ & \\
\hline \multicolumn{5}{|c|}{$\begin{array}{l}\text { Probability of adverse events within each stratum was estimated via the Kaplan-Meier estimator. When the risk difference or risk ratio was } \\
\text { calculated, the urban cohort was used as the reference group. } \\
\text { ^p values for Wilcoxon trend tests. } \\
\text { ED, emergency department. }\end{array}$} \\
\hline
\end{tabular}

This study has several limitations. NHIRD did not contain information of patients' actual disease severity or duration of illness, both of which may affect patients' mental health needs after discharge. Nevertheless, the psychotic patients included in this study were presumed to be in the early course of the illness and with symptoms severe enough to trigger hospitalisation. Hence, we used inequity to describe the disparities in psychiatric service utilisation between rural and urban patients with psychosis. However, uncertainty still exists whether the

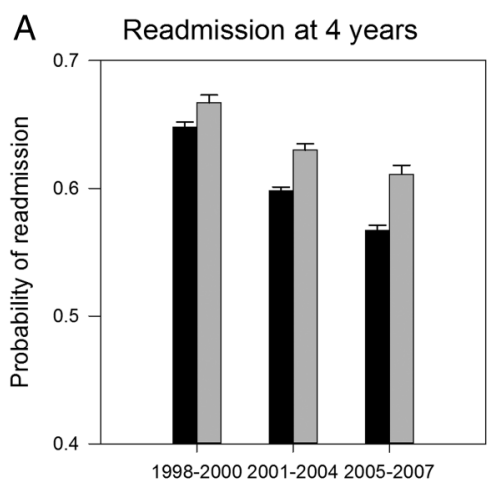

Year period

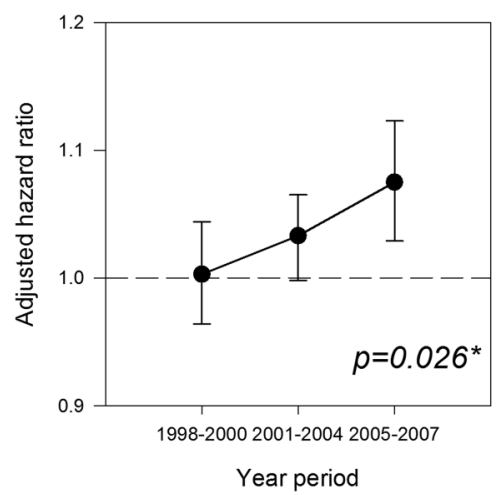

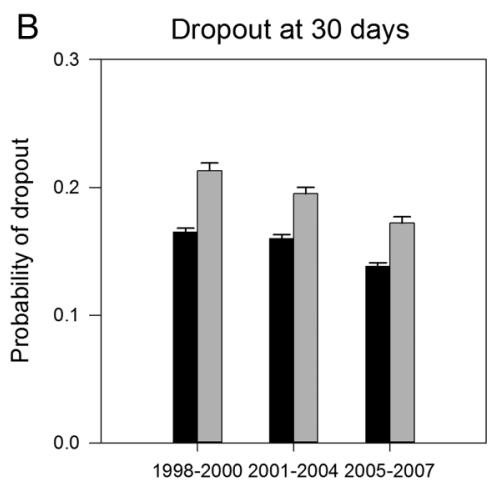

Year period

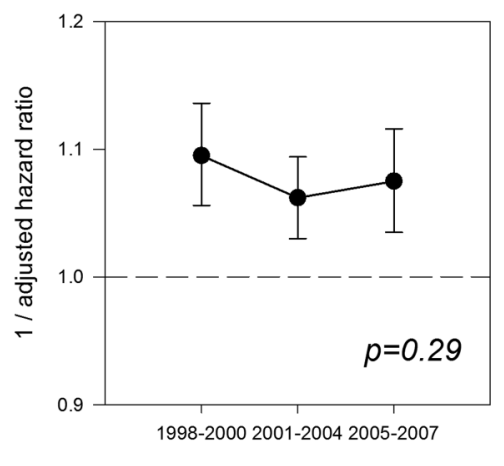

Year period

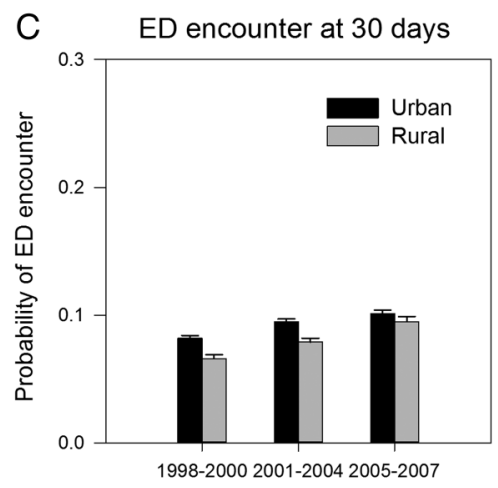

Year period

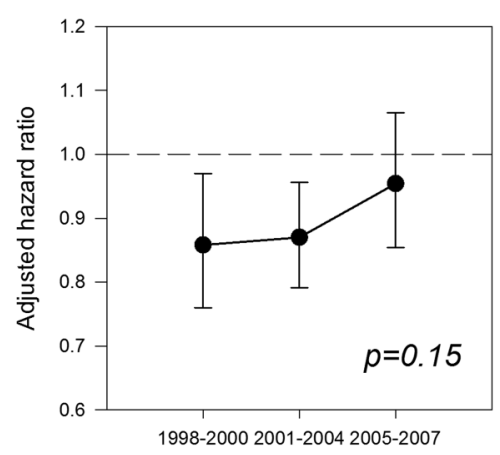

Year period

Figure 1 Temporal changes of risks for adverse events in relation to residence: (A) readmission at 48 months; (B) loss to follow-up at 30 days; and (C) ED encounters at 30 days. The upper panels are absolute risks in the urban and rural cohorts, respectively. The lower panels are relative risks presented as HRs or the reciprocals of HRs with the urban cohort as the reference group. $p$ Values for residence-by-period interaction are also presented. A relative risk of moving away from the value of one over time implies that that urban-rural inequality had been increasing over time, and vice versa. HRs are additionally adjusted for age, gender, diagnosis, hospital type, length of stay and economic status. ED, emergency department. 
rural and urban patients whom we identified had a similar severity or were in a similar point in the illness trajectory. However, urban and rural patients' presumed duration of illness in the database did not differ. Information regarding patients' premorbid adjustment and their carers' attitude, both being established risk factors for relapse in first-episode psychosis, ${ }^{33}$ was not available. Patients' residence information analysed in this study was presumed, not actual. Besides, psychotic diagnoses in the claims data lack further clinical validation. Our proxy classification about the patient's economic status may not be accurate enough to reflect the patient's actual household wealth. It should also be noted that readmission is closely related, but not identical, to relapse. ${ }^{34}$ Since the analyses only include patients who had been admitted to the psychiatric wards for the management of psychosis, the findings might not be generalised to patients with other mental illness or psychotic patients treated as outpatients throughout their illness course.

Finally, when interpreting our results, the influence of social determinants on health ${ }^{35}$ should be taken into consideration. Evidence suggests that the mental health of Taiwan's general population has worsened in parallel with macrosocial changes, such as the trends of national unemployment and divorce rates, during the 10-year study period. $^{36}$ These changes may also affect the mental health needs of our study population. We can only adjust for each patient's economic status, which also reflects employment status, in the regression models. However, such efforts might not be sufficient to adjust for the influence of social determinants.

\section{Explanation and meaning of this study}

By offering a glimpse of the multifaceted socioeconomic inequity, our finding on the urban-rural inequity in psychiatric service use adds to the growing literature on the potential limitations of UHC. An early review suggested that a mere expansion of health coverage is unlikely to reduce socioeconomic differences in health outcomes. ${ }^{37}$ More recently, other lesser-known pitfalls of UHC were pointed out, such as causing a paradoxical widening in health inequity and being taken as an excuse for policymakers to actively overlook the specific needs of disadvantaged people. ${ }^{38}$

In Taiwan, rural patients with psychosis represent a particularly disadvantaged but long-ignored patient group. Greater risks of readmission, a longer length of stay $^{13}$ and a lesser likelihood of staying in treatment ${ }^{39}$ have all reduced their chances to live in the community and participate in social life. Unfortunately, Taiwan's Mental Health Act has not been revised until 2007 to facilitate the development of community-based care, including prevention and rehabilitation, for psychiatric patients. Moreover, resources are still insufficient, unevenly distributed and under-developed, despite the fact that the investment in community-based treatment and rehabilitation has shown an increasing trend in the
NHI era. ${ }^{40}$ Although we did not actually measure patients' utilisation of community-based services in this study, we believe that community-based facilities in Taiwan are still under-developed, especially in rural areas. To further reduce the readmission rate of rural patients, resource reallocation from hospital-based to community-based care as well as improving delivery of psychiatric service would be essential steps.

Our findings have deepened the understanding of pros and cons of different healthcare systems. Compared with other systems, Taiwan's NHI is unique in that it is 'free': medical services are highly affordable to ordinary people, and people can freely choose their own service providers without the restraint of a referral system. The advantage of Taiwan's NHI is that people with greater health needs may obtain more healthcare than those with lesser needs, that is, greater vertical equity, ${ }^{19}$ which has led some to claim that Taiwan's NHI serves as a reference model for health reform in other countries. ${ }^{41}$ Unfortunately, our findings suggest that Taiwan's NHI did not decrease readmissions in rural patients as much as it did in urban patients. In other words, horizontal equity (defined as equal treatment for equal needs) related to urban-rural residence has worsened. Although we were unable to examine vertical equity in this study, we did not question the improved vertical equity among Taiwan's patients with psychosis. What we question, however, is the untested assumption that improvement in vertical equity can lead to improvement in horizontal equity.

The magnitude of urban-rural disparities in service utilisation found in this study might not be as great as in other countries with UHC. For example, in Taiwan, rural patients with psychosis had a $7 \%$ lower chance to see a psychiatrist after discharge than urban patients, whereas in Ontario, Canada, formerly hospitalised adolescents who lived in rural areas had an $18 \%$ lower chance to receive aftercare (provided by psychiatrists or primary care physicians) than urban adolescents. ${ }^{10}$ One possible explanation is that the contrast in the provision of mental health service between rural and urban areas in Taiwan, one of the most densely populated countries in the world, is less than that in Canada.

In the context of Taiwan's NHI, three previous studies had examined the temporal trends of urban-rural disparities, including the incidence of ruptured appendicitis, ${ }^{42}$ utilisation of hospice care ${ }^{30}$ and the elderly's utilisation of health services. ${ }^{14}$ A positive ${ }^{42}$ or mixed effect $^{1430}$ of NHI on urban-rural equity was found. In this study, urban-rural inequity in outpatient and ED services has been stable, and inequity in hospital admission has widened. The discrepancy in these findings may result from the different requirements of health conditions (acute or long-term care; general or specialty care; single-disciplinary or multidisciplinary care) and the different nature of dependent variables (outpatient or inpatient service; service utilisation or health status). As a complex disease, psychosis requires long-term, 
specialty and multidisciplinary care, while appendicitis only requires acute surgical treatment. It is probable that NHI may help to decrease the urban-rural gap of general medical and surgical services, but may not help to decrease the urban-rural gap of specialty or multidisciplinary services. Moreover, different results related to service types may be caused by patients' health status when they use the service. Patients with psychosis may have outpatient visits or ED treat-and-release encounters when their mental conditions are less severe. It is therefore probable that the more serious the illness, the greater the difficulty in decreasing the urban-rural inequity in the management of the illness.

Some merits of Taiwan's health system as revealed in this study should still be highlighted. In our data, over $40 \%$ of patients with psychosis were living on the lowest income. Thanks to NHI, even these patients can afford mental health services and medications, including the expensive second-generation antipsychotics, which help improve patients' medical adherence ${ }^{43}$ and prevent more hospitalisations ${ }^{44}$ than first-generation antipsychotics do. In other words, the national trends towards fewer readmissions and early dropouts in this study could be partially attributable to NHI. Limited by the study design, however, we cannot determine the extent to which NHI could explain such progress. Other concurrent changes, like advances in psychiatric practice and increased supply of mental health professionals, may also contribute to the overall progress. In addition, efforts in deinstitutionalisation have shown some results. In our data, the percentage of long-stay admissions decreased from $13.6 \%$ in $1998-2000$ to $8.8 \%$ in 2005-2007. Therefore, the national trend towards an increase in early ED encounters after discharge was probably an inevitable consequence. Fortunately, the risk of readmission did not elevate, meaning that an actual revolving door phenomenon did not happen.

\section{Unanswered questions and future research}

Our findings about the progressively worsening urbanrural health inequity in Taiwan will need further replication in other psychiatric or medical conditions. Moreover, some facets of inequity, such as difference in the practices of pharmacological and psychosocial interventions, should be examined in order to provide directions for future policy interventions in Taiwan.

Mental illness and its treatment have received little attention during the realisation of UHC, and relevant studies, especially in middle income and low income countries, are surprisingly few. We therefore call for more empirical studies to investigate the mental health issue in psychiatric patients or general populations in the context of UHC.

\section{CONCLUSIONS}

In a health system designated for equity, urban-rural inequity in mental service utilisation remains difficult to resolve for patients with psychosis. Our findings highlight the importance of regular equity-oriented monitoring of the health system. Moreover, our findings highlight the difficulty in the management of patients facing dual challenges from psychosis and distance barriers. Besides health coverage expansion and removal of financial barriers, additional policy interventions on resource allocation, service delivery and quality of care are needed to improve the outcome of rural-dwelling patients with psychosis.

\section{Author affiliations}

${ }^{1}$ Master of Public Health Degree Program, College of Public Health, National Taiwan University, Taipei, Taiwan

2Department of Psychiatry, Shin Kong Wu Ho-Su Memorial Hospital, Taipei, Taiwan

${ }^{3}$ Institute of Epidemiology and Preventive Medicine, College of Public Health, National Taiwan University, Taipei, Taiwan

${ }^{4}$ Health Data Research Center, National Taiwan University, Taipei, Taiwan

${ }^{5}$ Institute of Health Policy and Management, College of Public Health, National Taiwan University, Taipei, Taiwan

${ }^{6}$ Department of Psychiatry, College of Medicine, National Taiwan University, Taipei, Taiwan

${ }^{7}$ Department of Psychiatry, National Taiwan University Hospital, Taipei, Taiwan

Acknowledgements The authors thank Ms Chia-Hsien Yang (project manager) in the Health Data Research Center, National Taiwan University, for her assistance in the conduct of the project.

Contributors WJC had full access to all the data in the study and takes responsibility for the integrity of the data and the accuracy of the data analysis. C-LC and WJC were responsible for the study concept and design. C-LC, P-CC, L-YH, P-HK, Y-CT, C-CL and WJC were responsible for the acquisition, analysis and interpretation of data. C-LC and WJC were responsible for the drafting of the manuscript. C-LC, P-CC, P-HK, Y-CT, C-CL and WJC were responsible for the critical revision of the manuscript for important intellectual content. C-LC, L-YH and WJC were responsible for the statistical analysis. WJC was responsible for obtained funding. L-YH, P-CC and WJC were responsible for the administrative, technical and material support. WJC carried out the study supervision.

Funding This study was supported by a grant from the National Science Council (NSC101-3114-Y-002-003) to Prof. Ming-Je Tang and a grant from the "Aim for the Top University Project" of National Taiwan University, funded by the Ministry of Education of Taiwan, to WJC (103RH00240).

\section{Competing interests None declared.}

Ethics approval The Institutional Review Board of the National Taiwan University Hospital approved this study.

Provenance and peer review Not commissioned; externally peer reviewed.

Data sharing statement No additional data are available.

Open Access This is an Open Access article distributed in accordance with the Creative Commons Attribution Non Commercial (CC BY-NC 4.0) license, which permits others to distribute, remix, adapt, build upon this work noncommercially, and license their derivative works on different terms, provided the original work is properly cited and the use is non-commercial. See: http:// creativecommons.org/licenses/by-nc/4.0/

\section{REFERENCES}

1. Perala J, Suvisaari J, Saarni SI, et al. Lifetime prevalence of psychotic and bipolar I disorders in a general population. Arch Gen Psychiatry 2007;64:19-28.

2. Saxena S, Thornicroft G, Knapp M, et al. Resources for mental health: scarcity, inequity, and inefficiency. Lancet 2007;370:878-89.

3. Evans DB, Etienne C. Health systems financing and the path to universal coverage. Bull World Health Organ 2010;88:402.

4. Hosseinpoor AR, Bergen N, Koller T, et al. Equity-oriented monitoring in the context of universal health coverage. PLoS Med 2014;11:e1001727. 
5. Kolappa K, Henderson DC, Kishore SP. No physical health without mental health: lessons unlearned? Bull World Health Organ 2013;91:3-3A.

6. Whiteford HA, Degenhardt L, Rehm J, et al. Global burden of disease attributable to mental and substance use disorders: findings from the Global Burden of Disease Study 2010. Lancet 2013;382:1575-86.

7. Eaton J, McCay L, Semrau M, et al. Scale up of services for mental health in low-income and middle-income countries. Lancet 2011;378:1592-603.

8. Steele LS, Glazier RH, Lin E. Inequity in mental health care under Canadian universal health coverage. Psychiatr Serv 2006:57:317-24.

9. Houle J, Beaulieu MD, Lespérance F, et al. Inequities in medical follow-up for depression: a population-based study in Montreal. Psychiatr Serv 2010;61:258-63.

10. Carlisle C, Mamdani M, Schachar R, et al. Predictors of psychiatric aftercare among formerly hospitalized adolescents. Can J Psychiatry 2012;57:666-76.

11. Durbin A, Moineddin R, Lin E, et al. Examining the relationship between neighbourhood deprivation and mental health service use of immigrants in Ontario, Canada: a cross-sectional study. BMJ Open 2015;5:e006690.

12. Hwu HG, Yeh EK, Chang LY. Prevalence of psychiatric disorders in Taiwan defined by the Chinese Diagnostic Interview Schedule. Acta Psychiatr Scand 1989;79:136-47.

13. Tseng KC, Hemenway D, Kawachi I, et al. Travel distance and the use of inpatient care among patients with schizophrenia. Adm Policy Ment Health 2008;35:346-56.

14. Liao PA, Chang HH, Yang FA. Does the universal health insurance program affect urban-rural differences in health service utilization among the elderly? Evidence from a longitudinal study in Taiwan. $J$ Rural Health 2012;28:84-91.

15. Wu AC, $\mathrm{Hu} \mathrm{YH}$. Many ways to health: a study of 2,000 rural and urban Taiwan families. Am J Chin Med 1980;8:313-30.

16. Hwu HG, Rin H, Chen CZ, et al. A study on the personal-familial and clinical data of psychiatric inpatients in Taiwan. Chin Psychiatry 1995;9:16-31.

17. Cheng TM. Reflections on the 20th anniversary of Taiwan's single-payer national health insurance system. Health Aff (Millwood) 2015;34:502-10.

18. Cheng SH, Chiang TL. The effect of universal health insurance on health care utilization in Taiwan. Results from a natural experiment. JAMA 1997;278:89-93.

19. Wang SI, Yaung CL. Vertical equity of healthcare in Taiwan: health services were distributed according to need. Int $J$ Equity Health 2013;12:12

20. Wang $\mathrm{H}$, Yip W, Zhang $\mathrm{L}$, et al. The impact of rural mutual health care on health status: evaluation of a social experiment in rural China. Health Econ 2009;18(Suppl 2):S65-82.

21. Liang Y, Lu P. Medical insurance policy organized by Chinese government and the health inequity of the elderly: longitudinal comparison based on effect of New Cooperative Medical Scheme on health of rural elderly in 22 provinces and cities. Int J Equity Health 2014:13:37.

22. Addington DE, McKenzie E, Wang J. Validity of hospital admission as an outcome measure of services for first-episode psychosis. Psychiatr Serv 2012;63:280-2.

23. Beadles CA, Ellis AR, Lichstein JC, et al. First outpatient follow-up after psychiatric hospitalization: does one size fit all? Psychiatr Serv 2015:66:364-72.

24. Vashi AA, Fox JP, Carr BG, et al. Use of hospital-based acute care among patients recently discharged from the hospital. JAMA 2013;309:364-71.
25. Bromet EJ, Schwartz JE, Fennig S, et al. The epidemiology of psychosis: the Suffolk County Mental Health Project. Schizophr Bull 1992;18:243-55.

26. Strakowski SM, Keck PE Jr, McElroy SL, et al. Twelve-month outcome after a first hospitalization for affective psychosis. Arch Gen Psychiatry 1998;55:49-55.

27. Selten JP, Veen N, Feller W, et al. Incidence of psychotic disorders in immigrant groups to The Netherlands. Br J Psychiatry 2001:178:367-72.

28. Lin $\mathrm{MH}$, Yang $\mathrm{AC}$, Wen $\mathrm{TH}$. Using regional differences and demographic characteristics to evaluate the principles of estimation of the residence of the population in National Health Insurance Research Databases (NHIRD). Taiwan J Public Health 2011;30:347-61.

29. Liu CY, Hung YT, Chuang YL, et al. Incorporating development stratification of Taiwan townships into sampling design of large scale health interview survey. J Health Manag 2006;4:1-22.

30. Lin $\mathrm{YH}$, Chen $\mathrm{YC}$, Tseng $\mathrm{YH}$, et al. Trend of urban-rural disparities in hospice utilization in Taiwan. PLoS ONE 2013;8:e62492.

31. Lin $\mathrm{CH}$, Chen WL, Lin CM, et al. Predictors of psychiatric readmissions in the short- and long-term: a population-based study in Taiwan. Clinics (Sao Paulo) 2010;65:481-9.

32. Kirkbride JB, Croudace T, Brewin J, et al. Is the incidence of psychotic disorder in decline? Epidemiological evidence from two decades of research. Int J Epidemiol 2009;38:1255-64.

33. Alvarez-Jimenez M, Priede A, Hetrick SE, et al. Risk factors for relapse following treatment for first episode psychosis: a systematic review and meta-analysis of longitudinal studies. Schizophr Res 2012;139:116-28.

34. Addington DE, Patten SB, McKenzie E, et al. Relationship between relapse and hospitalization in first-episode psychosis. Psychiatr Serv 2013;64:796-9.

35. Putnam S, Galea S. Epidemiology and the macrosocial determinants of health. J Public Health Policy 2008;29:275-89.

36. Fu TS, Lee CS, Gunnell D, et al. Changing trends in the prevalence of common mental disorders in Taiwan: a 20-year repeated cross-sectional survey. Lancet 2013;381:235-41.

37. Adler NE, Boyce WT, Chesney MA, et al. Socioeconomic inequalities in health: no easy solution. JAMA 1993;269:3140-5.

38. Gwatkin DR, Ergo A. Universal health coverage: friend or foe of health equity? Lancet 2011;377:2160-1.

39. McCarthy JF, Blow FC, Valenstein M, et al. Veterans Affairs Health System and mental health treatment retention among patients with serious mental illness: evaluating accessibility and availability barriers. Health Serv Res 2007;42:1042-60.

40. Wang JY. Review and outlook: the development of mental health policy and services in Taiwan. J Asian Public Policy 2009;2:111-7.

41. Yeh CC. Access and cost: what the U.S. health care system canlearn from other countries: hearing before the Subcommittee on Primary Care and Aging, Senate Committee on House, Education, Labor and Pensions (11 Mar 2014). http://www.help.senate.gov/imo/ media/doc/Yeh1.pdf (assessed 15 Nov 2015).

42. Huang N, Yip W, Chang HJ, et al. Trends in rural and urban differentials in incidence rates for ruptured appendicitis under The National Health Insurance in Taiwan. Public Health 2006;120:1055-63.

43. Wu CS, Lin YJ, Feng J. Trends in treatment of newly treated schizophrenia-spectrum disorder patients in Taiwan from 1999 to 2006. Pharmacoepidemiol Drug Saf 2012;21:989-96.

44. Gau SS, Chung CH, Gau CS. A pharmacoeconomic analysis of atypical antipsychotics and haloperidol in first-episode schizophrenic patients in Taiwan. J Clin Psychopharmacol 2008;28:271-8. 